\title{
Muir-Torre Syndrome
}

National Cancer Institute

\section{Source}

National Cancer Institute. Muir-Torre Syndrome. NCI Thesaurus. Code C84905.

A usually autosomal dominant inherited neoplastic syndrome caused by mutations in the hMSH-2 and hMLH-1 genes. It is characterized by the presence of sebaceous skin tumors (adenoma or carcinoma), and internal organ malignant tumors, usually of the gastrointestinal or genitourinary tract. 